\section{Emotion Regulation}

Emotion regulation (ER) is associated with description of emotional experiences and responses to them (Diefenbach, Tolin, Meunier, \& Worhunsky, 2008). ER is defined as ability to smooth and manage the emotional experiences for adaptation and enhancing psychological functioning (Jacob, Morelen, Suveg, Brown Jacobsen, \& Whiteside, 2012).

\section{Emotion Regulation Strategies}

\section{Emotional Avoidance}

Suppression

Distraction

Denial

Rationalization

Substance Use

Dissociation

ER descriptions in some sources emphasize the ability to control negative emotions and reduce the level of arousal. On the other hand, some sources emphasize to experience, clarification and response to all emotions (Gratz \& Roemer, 2004). The explanation in the second group points to awareness and understanding of emotions. Thus, it develops the skills of monitoring,

experiencing, accepting and regulating both positive and negative emotions (Gratz \& Roemer, 2004).

Impaired decision-making mechanisms and goal-directed behaviors

Worry

Unipolar depression

Generalized anxiety disorder

Eating disorders

Borderline personality disorder

Internalization symptoms

Body focused repetitive behaviors

\section{Obsessive Compulsive Disorder}

Individuals with OCD experience more anxiety than healthy individuals when they encounter with disorder-related stimuli and direct their attention to disorder-related stimuli more quickly (van den Heuvel et al., 2004).

They feel compelled to make compulsions to reduce the anxiety associated with obsessions (Paul, Simon, Endrass, \& Kathmann, 2016).

Individuals with OCD cannot resist compulsions because they have so much anxiety that they cannot cope (Taylor \& Liberzon, 2007).

Therefore, it was thought that excessive anxiety related to stimulants may be related to disorders in ER mechanism and compulsions may be maladaptive ER strategies.

\section{Models to explain the relationship between OCD and ER}

\section{- Coleman, Pieterefesa, Holaway, Coles, \& Heimberg, 2011}

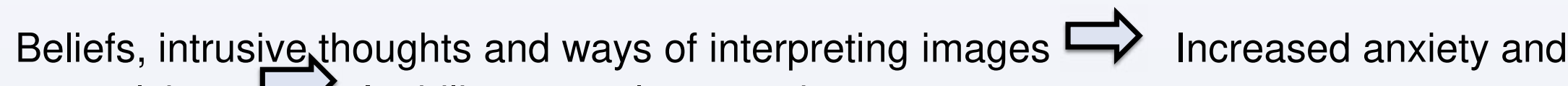
compulsions $\Rightarrow$ Inability to regulate emotions

- Coles, Schofield, \& Pieterefesa, 2006

Tendency to avoid changes and being exposed to excessive protection as a child $\vec{\longrightarrow}$ Deficiencies in dealing with difficult situations, problem solving and coping with accompanying negative emotions $\Rightarrow$ Becoming not be able to understand emotions $\Rightarrow$ Discomfort $\vec{\square}$ Avoidance of emotion triggering conditions in the future

It is suggested that the deficiencies in recognizing and understanding of emotions lead the individual to make an effort not to experience these emotions and use maladaptive ER strategies (rumination, suppression, avoidance) when he/she has to experience the emotions.

\section{- Calkins, Berman, \& Wilhelm, 2013}

Pay attention to the negativity and potential consequences of intrusive thoughts $\vec{\Rightarrow}$ Anxiety and anxiety suppression attempts $\Rightarrow$ Lack of confidence in the individual's ability to regulate moods that stress him/her $\overrightarrow{ }$ Continuing to use maladaptive coping strategies

The use of compulsions prevents one from confronting stressful emotions and prevents the entry of inaccurate information.

\section{Clinical Implications}

- ER skills can be taught to the patient before starting exposure therapies (de la Cruz et al., 2013).

- Cognitive Behavioral Therapies for OCD are used to teach ER strategies to help cognitive restructuring (De Wit et al., 2015).

- Conscious awareness and Acceptance and Commitment Therapy (ACT) emphasizes the acceptance and awareness of all thought and emotional experiences, regardless of value, intensity and perceived benefit.

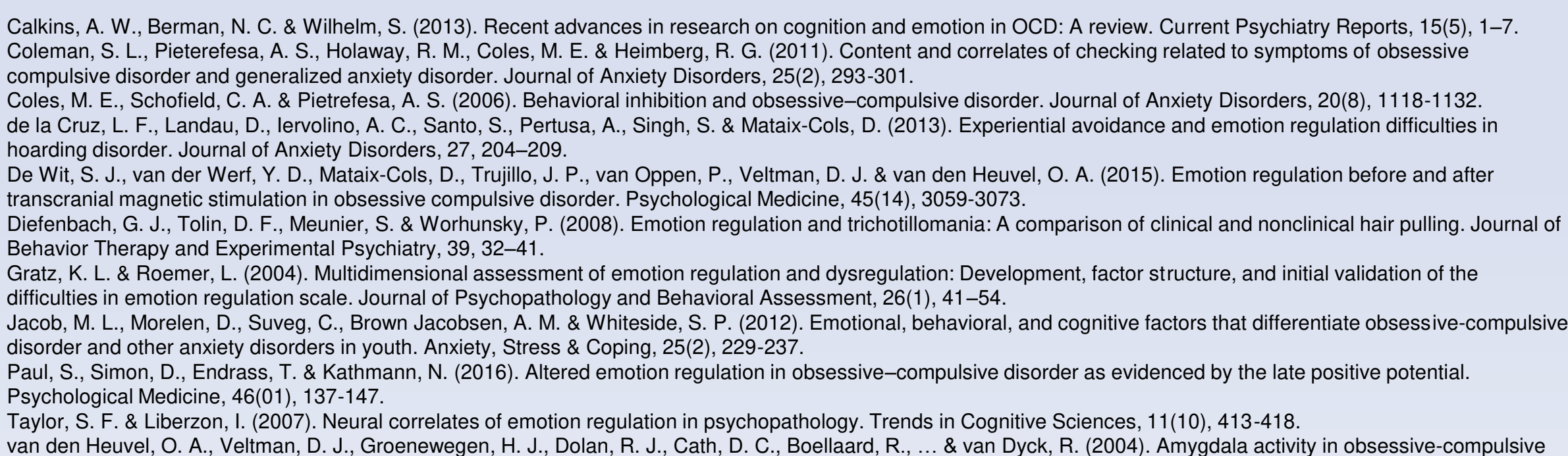

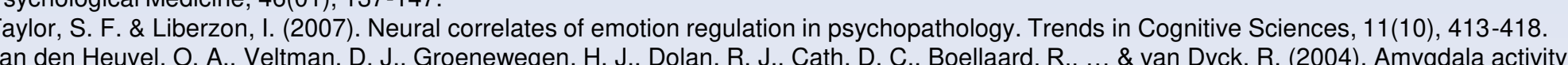
van den Heuvel, O. A. Veltman, D. J., Groenewegen, H. J., Dolan, R. J, Cath, D. C., Boellaard, R.,...\& \&an Dyck, R. (2004). Amygdala activity in obsessive-compulsive
disorder with contamination fear: A study with oxygen-15 water positron emission tomography. Psychiatry Research, 132, 225-237. 